\title{
Trajectories of the relationships of physical activity with body composition changes in older men: the MrOS study
}

\author{
Deepika R. Laddu ${ }^{1 *}$ (D) Peggy M. Cawthon ${ }^{2,3}$, Neeta Parimi ${ }^{2}$, Andrew R. Hoffman ${ }^{4}$, Eric Orwoll ${ }^{5}$, Iva Miljkovic ${ }^{6}$, \\ Marcia L. Stefanick ${ }^{7}$ and for the Osteoporotic Fractures in Men Study Research Group
}

\begin{abstract}
Background: Excess adiposity gains and significant lean mass loss may be risk factors for chronic disease in old age. Long-term patterns of change in physical activity (PA) and their influence on body composition decline during aging has not been characterized. We evaluated the interrelationships of PA and body composition at the outset and over longitudinal follow-up to changes in older men.

Methods: Self-reported PA by the Physical Activity Scale for the Elderly (PASE), clinic body weight, and whole-body lean mass (LM) and fat mass, by dual-energy $x$-ray absorptiometry (DXA), were assessed in 5964 communitydwelling men aged $\geq 65$ years at baseline (2000-2002) and at two subsequent clinic visits up until March 2009 (an average 4.6 and 6.9 years later). Group-based trajectory modeling (GBTM) identified patterns of change in PA and body composition variables. Relationships of PA and body composition changes were then assessed.

Results: GBTM identified three discrete trajectory patterns, all with declining PA, associated primarily with initial PA levelshigh-activity (7.2\% of men), moderate-activity (50.0\%), and low-activity (42.8\%). In separate models, GBTM identified eight discrete total weight change groups, five fat mass change groups, and six LM change groups. Joint trajectory modeling by PA and body composition group illustrated significant declines in total weight and LM, whereas fat mass levels were relatively unchanged among high-activity and low-activity-declining groups, and significantly increased in the moderate-activity-declining group.
\end{abstract}

Conclusion: Although patterns of change in PA and body composition were identified, groups were primarily differentiated by initial PA or body composition rather than by distinct trajectories of change in these variables.

Keywords: Physical activity, Lean mass, Fat mass, Older men, Trajectories

\section{Background}

Significant weight loss, particularly substantial lean mass loss [1], and fat mass gain have been identified as risk factors for chronic diseases in aging men [1-4]. Muscle mass has been reported to decline $1-3 \%$ per decade in men starting by age 30 and $2-3.3 \%$ per decade after age $60[3,5]$. Progressive skeletal muscle loss accompanied by reduced muscle strength and declining functional performance $[4,6,7]$, referred to as sarcopenia [6], leads

\footnotetext{
* Correspondence: dladdu@uic.edu

'Department of Physical Therapy, University of Illinois at Chicago, Chicago,

Illinois 60612, USA

Full list of author information is available at the end of the article
}

to frailty and physical and mobility disabilities. Concomitant gains in fat mass also contribute to the reduced physical function and quality of life. [8].

Participation in regular physical activity (PA) has been shown to promote favorable changes in body composition, including prevention of lean mass loss and reduced fat mass gain [9-12]. Yet, PA generally decreases with age and only $37.8 \%$ of men aged $65-74$ years report participating in the recommended minimum of 30 minutesmin of moderate-intensity physical activity on 5 or more days per week [13, 14]. The relationship of declining PA to lean mass loss or inability to retain lean mass may play an 
important role in the functional limitations that arise with aging.

Epidemiological evidence supports the hypothesis that higher regular PA and/or increasing PA levels attenuate total and fat weight gain through middle age $[15,16]$. Substantial literature also suggests that agerelated changes in body composition precede the gradual decline in PA as men age $[1,11,17]$. We are unaware of an assessment of both PA and body-composition changes or trajectories of these changes over an extended period in older men.

The long-term follow-up of the Osteoporotic Fractures in Men Study (MrOS) cohort provided the opportunity to apply innovative, methodological advances in the study of developmental trajectories [18, 19] to assess patterns of change in PA behavior during aging, taking into consideration baseline (routine) PA. Using a finite mixture modeling strategy to classify distinctive groups of older men based on similarities between their baseline and changes in PA, we estimated the proportion of men who demonstrated distinct patterns of PA change over an average 6.9 year follow-up. By relating the probability of group membership to individual's PA, we can evaluate how PA changes may relate to changes in body composition (i.e., fat and lean masses) patterns as older men age. Finally, we applied joint (dual) trajectory models to analyze the correlation between the PA and body composition trajectories that were evolving contemporaneously as men aged, and to describe the development of the PAbody composition relationship over time. Additionally, multi-trajectory models, which were used to illustrate distinct patterns in the joint trajectories of PA and body composition, provides a compact approach for summarizing the within-individual correspondence of longitudinal data of PA and body composition measurements which other methods are unable to capture.

\section{Methods \\ Participants}

From March 2000 through April 2002, 5994 men, aged 65 years and older, enrolled in and completed baseline assessments in the multicenter MrOS study [20]. Recruitment was primarily through community-based mailing lists to six-geographic regions in the United States: Birmingham, AL; Minneapolis, MN; Palo Alto, CA; Pittsburgh, PA; Portland, OR; and San Diego, CA [20, 21]. Exclusions included bilateral hip replacement or inability to walk without the assistance of another person. A second and third clinic visit (V2 and V3) were conducted an average of 4.6 years and 6.9 years after baseline, respectively. Men who died or terminated were included in the trajectory building as long as they contributed at least one visit with PASE data before death or termination. For example, if they contributed PASE data at V1 and V2 and died after V 2, then their V1 and V2 values contributed to the trajectory model. Thus, this report of trajectory models was restricted to 5964 men who provided selfreport data for physical activity (PA) by the Physical Activity Scale for the Elderly (PASE) and had a DXA measurement at one or both follow-up visits (Additional file 1: Figure S1). However, in models reporting absolute change in body composition trajectories relative to PA trajectories, men with loss to follow up due to deaths, termination or missing values were excluded from analyses. Thus, in absolute change models, only men who retuned for visit 3 and had non missing values for the body composition and PASE score were included change analyses. The total sample of men with visit 1 and visit 3 non missing measures of body weight: $n=3894$, lean mass: $n=3641$, and fat mass: $n=3641$. The institutional review board at each center approved the study protocol and written informed consent was obtained from all participants.

\section{Physical activity measurements}

PASE [22], developed specifically for community-dwelling older adults, was self-administered at baseline (V1), V2, and V3. PASE measures total, occupational, household, and leisure physical activities over the previous 7 days and asks about the intensity, frequency, and duration of a variety of activities, including walking; strenuous, moderate and light sports; muscle strength and endurance training; occupational activities, including standing or walking; lawn work and gardening; caring for another person; home repairs; and heavy and light housework. The frequency and duration of each activity is multiplied by an empirically derived item weight and summed to compute the total PASE score activity [22]. PASE scores, which have no units, provide a relative rather than absolute measure of PA levels. PASE has been previously validated with objective measures of physical activity [22-24], and has high test-retest reliability [22, 23].

\section{Assessment of weight, and body composition}

Body weight was recorded with a balance beam scale at all but the Portland MrOS clinical site, which used a digital scale. Whole-body fat mass and lean mass (LM) were measured from whole-body scans using fan beam DXA scanners (QDR $4500 \mathrm{~W}$, Hologic, Inc., MA), following a standardized procedures. All scan analyses were executed by certified DXA technicians. Reproducibility was ensured by use of a central quality control lab (San Francisco Coordinating Center). Quality assurance was carried out using a Hologic whole-body phantom, scanned repeatedly at each site to monitor reproducibility of measurements, and to calculate and apply appropriate correction factors to adjust for longitudinal drift in DXA measures. 


\section{Questionnaire data}

At baseline, participants reported their age, race/ethnicity, education, smoking status, alcohol use, and health status. Self-reported medical history data included history of physician diagnosis of diabetes mellitus, hypertension, coronary heart disease, congestive heart failure, chronic obstructive lung disease, and non-skin cancer.

\section{Statistical analysis}

Group-based trajectory modeling (GBTM) was applied using TRAJ software in STATA (v. 9.2) which identifies clusters of individuals following similar progressions of a specified phenotype over some measure of time (e.g.; age, follow up time). [19] GBTM are descriptive analyses that identify latent groups of individuals based on patterns (i.e., decline, incline, cyclic), while simultaneously accounting for unobserved heterogeneity in the data [18]. Data analysis proceeded through three steps. The maximum number of groups (trajectories) for each phenotype was decided a priori. A quadratic shape of the pattern of change per trajectory was specified for all models, as recommended by Jones et al. 2001 [18, 19]. The number of trajectories were then fit sequentially until the most parsimonious model was determined by either the maximum number of groups decided by the Bayesian Information Criterion (BIC) or a priori. Briefly, the best fit model and number of trajectory groups were determined using the $2 \Delta \mathrm{BIC}>10$; such that subsequent complex models with an increased number of trajectories groups would not identify unique clusters of individuals. If the number of trajectories was greater than the maximum number of trajectories decided a priori then the models were fit with the number of trajectories decided prior to the analysis. After the final model was defined, mean posterior probabilities were calculated to ensure internal reliability of the model. In all models, an individual was assigned to a group based on his highest posterior group probability over the follow up. For generalizability, we only included specific groups comprised of at least $1 \%$ of the analytic cohort. The same model-fit criteria were repeated to determine trajectories of PA, total body weight and fat mass, and LM, over the maximum follow up, in separate models.

Joint trajectory modeling was employed to examine combinations of PA groups with each of the body composition variables (i.e., PA-weight, PA-fat mass, PA-LM) using similar model fit criteria. Baseline characteristics of participants were reported by PA trajectory groups as means, medians and proportions as appropriate, and differences between PA trajectory groups were tested using ANOVA and $x^{2}$ for continuous and categorical characteristics, respectively. Formal statistical tests, restricted to those who returned to visit 3 and had non missing measures at baseline and at visit 3 , were performed to determine:

1. whether changes from baseline (V1) to visit 3 in body composition measures were statistically significant from zero within each trajectory, without consideration of the PA trajectories. This test will determine if GBTM correctly (and significantly) described the trajectory's pattern (i.e., decline, incline, cyclic).

2. whether mean change in each body composition measure (computed [V3-V1] for each body composition trajectory) was significantly different with-in and across PA trajectories. Kruskal-Wallis $P$-values were reported due to slightly skewed body composition data. Tests of statistical significance were 2-tailed, with $\alpha=0.05$.

\section{Results}

\section{Subject characteristics}

PA trajectory patterns, all of declining PA, were identified by GBTM methods: high (baseline) activity, moderate activity, and low activity, representing $7.2 \%, 50.0 \%$, and $42.8 \%$, of the total analytic cohort, respectively. Descriptive statistics of the 5964 men in the cohort are presented in Table 1 by these PA (PASE score) trajectory groups. The average baseline age (SD) was 73.7 (5.9) years. The majority were Caucasian (89.4\%). A greater proportion of men in the low-activity-declining group reported having hypertension, diabetes, CVD and CHD and past-year fall history compared to men in the moderate or high activity baseline groups (all $P<.05$ ).

\section{Patterns of change in PA}

As noted above, the final models showed a decline in PA for all three trajectory groups ( $P$ for change not zero $<0.05)$; however, the decline in activity differed significantly among the three PA groups $(P<.0001)$ over the 6.9 year follow-up (Table 1, Fig. 1), with the greatest declines observed in men in the high-activity-declining group ( $P$ for pairwise difference between high-activitydeclining and low-activity-declining <.001; $P$ for pairwise difference between high-activity-declining and moderateactivity-declining <.001).

\section{Individual patterns of change in body weight, LM, and fat mass}

When changes of body weight, total body fat mass and LM were analyzed individually, there were eight body weight change groups, five fat mass change groups and six LM change groups in the most parsimonious models. Changes from baseline to visit 3 were shown to be declines in almost all eight weight groups and all six LM groups, with greater declines in body weight and LM 
Table 1 Baseline Characteristics by Physical Activity Groups in 5964 MrOS Men

\begin{tabular}{|c|c|c|c|c|c|}
\hline \multirow[b]{2}{*}{ Characteristics } & \multicolumn{3}{|c|}{ PA trajectories (PASE score) } & \multirow[b]{2}{*}{$\begin{array}{l}\text { Whole cohort } \\
(N=5964)\end{array}$} & \multirow[b]{2}{*}{$P$} \\
\hline & $\begin{array}{l}\text { Low-activity-declining } \\
(N=2555)\end{array}$ & $\begin{array}{l}\text { Moderate- activity- declining } \\
(N=2977)\end{array}$ & $\begin{array}{l}\text { High- activity- declining } \\
(N=432)\end{array}$ & & \\
\hline Change in $\mathrm{PA}^{\mathrm{a}}$ & $-54.1(45.7)$ & $-58.5(50.1)$ & $-69.0(56.9)$ & $-57.6(49.1)$ & $<0.0001^{b}$ \\
\hline Mean age (y) & $73.5(5.8)$ & $73.8(5.9)$ & $73.5(6.2)$ & $73.7(5.9)$ & 0.21 \\
\hline Age Category & & & & & 0.37 \\
\hline$\leq 73$ yrs & $1373(53.7)$ & $1544(51.9)$ & $238(55.1)$ & 3155 (52.9) & \\
\hline$>73$ yrs & $1182(46.3)$ & $1433(48.1)$ & $194(44.9)$ & $2809(47.1)$ & \\
\hline Race/ethnicity (\%) & & & & & $<0.0001$ \\
\hline White & $2253(88.2)$ & $2699(90.7)$ & $382(88.4)$ & $5334(89.4)$ & \\
\hline African American & $134(5.2)$ & $100(3.4)$ & $8(1.9)$ & $242(4.1)$ & \\
\hline Asian & $96(3.8)$ & $83(2.8)$ & $12(2.8)$ & $191(3.2)$ & \\
\hline Hispanic & $42(1.6)$ & $59(2.0)$ & $25(5.8)$ & $126(2.1)$ & \\
\hline Other & $30(1.2)$ & $36(1.2)$ & $5(1.2)$ & $71(1.2)$ & \\
\hline College education or higher & $1364(53.4)$ & $1579(53.0)$ & $232(53.7)$ & $3175(53.2)$ & 0.95 \\
\hline Alcohol intake (drinks/wk) & & & & & 0.05 \\
\hline None & $957(37.5)$ & $1006(33.8)$ & $142(32.9)$ & 2105 (35.3) & \\
\hline Light & $641(25.1)$ & $789(26.5)$ & $112(25.9)$ & $1542(25.9)$ & \\
\hline Moderate/high & $952(37.3)$ & 1179 (39.6) & $178(41.2)$ & 2309 (38.8) & \\
\hline Smoking $(n, \%)$ & & & & & 0.0004 \\
\hline None/past smoker & 2441 (95.6) & $2891(97.1)$ & $426(98.6)$ & 5758 (96.6) & \\
\hline Current smoker & $113(4.4)$ & $86(2.9)$ & $6(1.4)$ & $205(3.4)$ & \\
\hline Self-reported health $(n, \%)$ & & & & & $<0.0001$ \\
\hline Very poor/poor/fair & $531(20.8)$ & $296(9.9)$ & $15(3.5)$ & $842(14.1)$ & \\
\hline Excellent/good & $2022(79.2)$ & $2681(90.1)$ & $417(96.5)$ & $5120(85.9)$ & \\
\hline Height, mean, cm & $174.4(6.9)$ & $174.0(6.7)$ & $173.6(6.5)$ & $174.1(6.8)$ & 0.03 \\
\hline Weight, mean, kg & $84.5(14.1)$ & $82.2(12.4)$ & $80.8(12.5)$ & $83.1(13.2)$ & $<0.0001$ \\
\hline $\mathrm{BMI}$, mean kg/m² & $27.8(4.1)$ & $27.1(3.5)$ & $26.8(3.7)$ & $27.4(3.8)$ & $<0.0001$ \\
\hline BMI Categories $\left(\mathrm{kg} / \mathrm{m}^{2}\right)$ & & & & & $<0.0001$ \\
\hline$<25$ & $640(25.1)$ & $861(28.9)$ & $138(31.9)$ & $1639(27.5)$ & \\
\hline $25-30$ & $1279(50.1)$ & $1540(51.8)$ & $231(53.5)$ & $3050(51.2)$ & \\
\hline$\geq 30$ & $636(24.9)$ & $574(19.3)$ & $63(14.6)$ & $1273(21.4)$ & \\
\hline History of CVD & 799 (31.3) & $747(25.1)$ & $77(17.8)$ & $1623(27.2)$ & $<0.0001$ \\
\hline History of CHD & $158(6.2)$ & $149(5.0)$ & $9(2.1)$ & $316(5.3)$ & 0.001 \\
\hline Self-reported cancer & $749(29.3)$ & $871(29.3)$ & $115(26.6)$ & $1735(29.1)$ & 0.501 \\
\hline Self -reported Hypertension & $1216(47.6)$ & $1214(40.8)$ & $133(30.8)$ & $2563(43.0)$ & $<0.0001$ \\
\hline Self- reported Diabetes & $362(14.2)$ & $268(9.0)$ & $18(4.2)$ & $648(10.9)$ & $<0.0001$ \\
\hline History of Fracture after 50 yrs & $585(22.9)$ & $655(22.0)$ & $110(25.5)$ & $1350(22.7)$ & 0.39 \\
\hline Fall in last year $(n, \%)$ & $582(22.8)$ & $581(19.5)$ & $94(21.8)$ & $1257(21.1)$ & 0.01 \\
\hline
\end{tabular}

Mean (SD) or proportion as shown

$B M I$ body mass index, $C H D$ coronary heart disease, $C V D$ cardiovascular disease, $P A$ physical activity

$P$-values indicates the overall difference among PA trajectories

${ }^{\text {a }}$ Change measured as change in PASE score from visit 1 to visit 3 .

bSignificance observed in PA change between high activity declining compared to moderate activity declining $(P<.001)$ or compared to low activity-declining groups $(P<0.001)$; ANOVA 


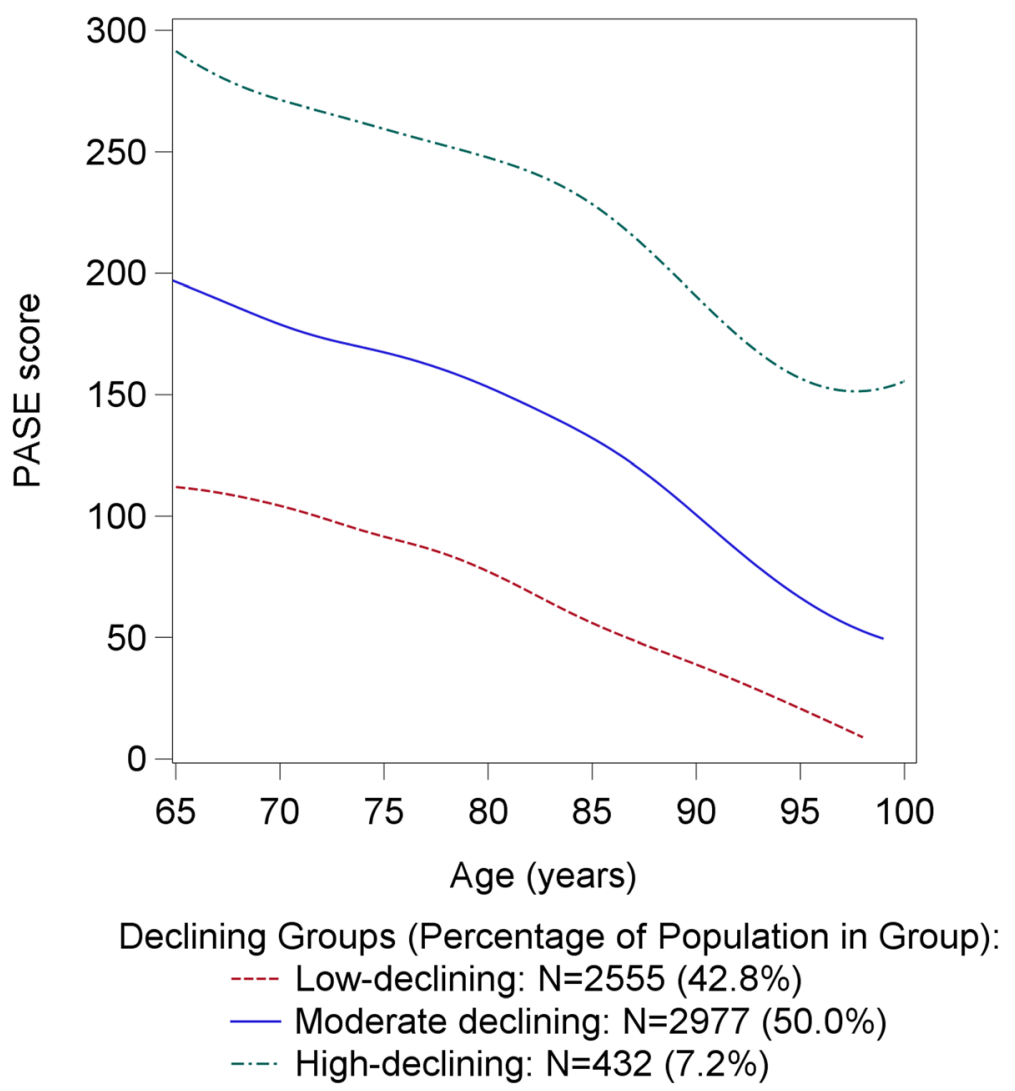

Fig. 1 PA trajectories by age; total $N=5964$; mean 6.9 year follow up. PA groups based on self-reported PA via PASE scores

among men with lower (trajectory 1 ) versus higher baseline weight or LM, respectively. Increases in fat mass were observed in two fat mass groups, and small, statistically significant declines were observed in one fat mass group from visit 1 to visit 3 (Additional file 1: Table S1, Fig. 2).

\section{Joint trajectory patterns of physical activity and body composition}

Figure $3 \mathrm{a}-\mathrm{c}$ graphically illustrates the joint trajectory models for changes in patterns of each body composition measure conditional on PA. For each joint trajectory model the most parsimonious models identified six trajectories for the body composition measure and three PA trajectories.

To determine whether body composition patterns differed significantly by PA trajectory, we computed the mean change (V3-V1) in the six groups of body weight, fat mass, LM groups, respectively, relative to the three declining PA trajectories (Table 2). We addressed whether: 1) changes in body composition measures from baseline to visit 3 were statistically significant from zero within each trajectory; 2) changes in body composition measures differed significantly within each PA trajectory; and, 3) changes in body composition measures from baseline to visit 3 were globally different across all three PA trajectories. Overall, body weight declined in most bodyweight/PA trajectory groups $\left(P_{\mathrm{V} 3-\mathrm{V} 1}<.05\right.$ for most $)$. Moreover, changes in body weight differed among the eighteen combinations of PA and body weight trajectories (overall ANOVA $P<.0001$ ). Within the low-activitydeclining and moderate-activity-declining groups, mean body weight changes by the six body weight trajectories were significantly different from one another $(P$ for ANOVA within activity declining groups <.0001). Conversely, there were no statistically significant differences in body weight change within the body weight trajectories for the high-activity-declining group (ANOVA within high activity group, $P=.24$ ).

Fat mass changes remained relatively unchanged (stable) across most fat mass/PA groups over time $\left(P_{\mathrm{V} 3-\mathrm{V} 1}>.05\right.$ for most); though significant increases in fat mass were observed in three fat mass trajectories in the moderateactivity-declining group $\left(P_{\mathrm{V} 3-\mathrm{V} 1}<.05\right)$. Changes in fat mass differed by the eighteen combinations of fat mass and PA patterns (overall ANOVA $P<.0001$ ). Within the low-activity-declining and moderate-activity-declining groups, significant differences in mean fat mass changes by the six fat mass trajectories were observed ( $P$ for ANOVA within activity-declining groups <.0001). 


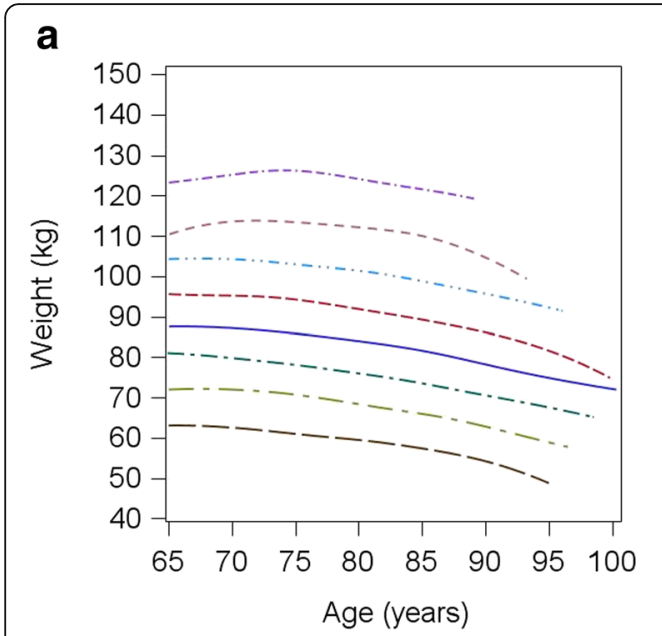

Trajectory Groups (Percentage of Population in Group):

- - Group 1: $N=305(5 \%) \quad---$ Group 5: N=950(16\%)

- - Group 2: $\mathrm{N}=1119(19 \%)$-... Group 6: $\mathrm{N}=437(8 \%)$

-.- Group 3: N=1583(26\%) - - - Group 7: N=151(3\%)

— Group 4: $N=1342(22 \%)$-..- Group 8: $N=77(1 \%)$

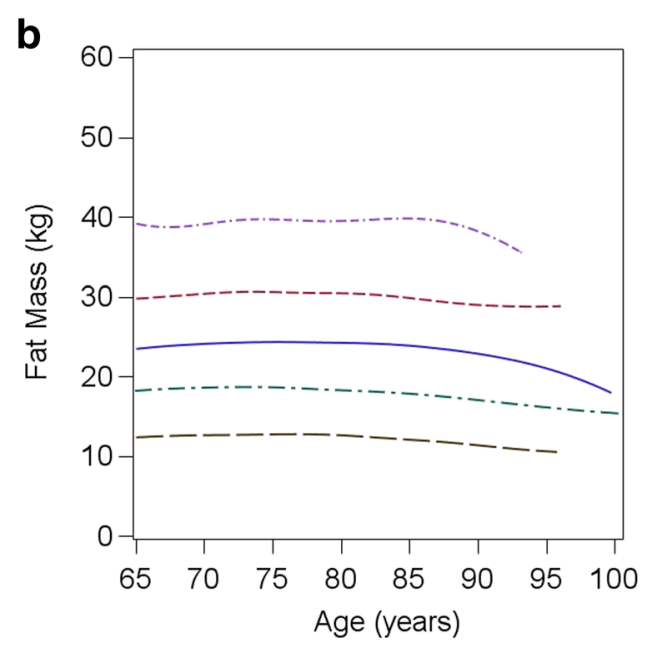

Trajectory Groups (Percentage of Population in Group):

- - Group 1: N=2203(36\%) ---- Group 4: N=917(16\%)

--- Group 2: N=1701(28\%) --.-- Group 5: N=286(5\%)

- Group 3: N=857(15\%)

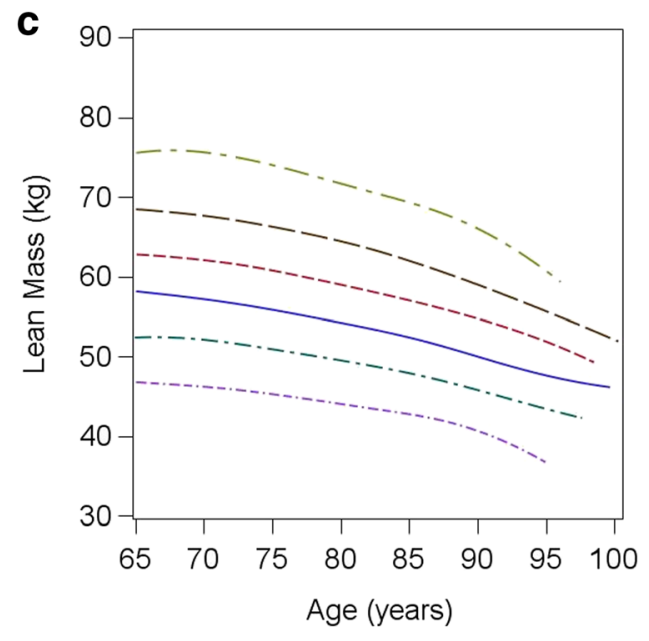

Trajectory Groups (Percentage of Population in Group): ---- Group 1: N=464(8\%) ---- Group 4: N=1361(23\%)

-.- Group 2: N=1378(23\%) - - Group 5: N=763(13\%)

- Group 3: N=1784(30\%) - - Group 6: N=214(4\%)

Fig. 2 a-c Trajectories of body weight (a), Total body fat mass (b), and Lean mass (c). Number of groups reflect most parsimonious model

However, the overall differences in fat mass change within the fat mass trajectories for the high-activity-declining group were not significant (ANOVA within high activity group, $P=.51$ ).

LM declined in most of the LM/activity pattern groups $\left(P_{\mathrm{V} 3-\mathrm{V} 1}<.05\right.$ for most). Changes in LM differed by the eighteen combinations of LM and activity patterns (overall ANOVA $P<.0001)$. Similar to the other body composition measures, there were differences in the mean changes in LM by LM trajectories within the low-activitydeclining and moderate-activity-declining groups, $(P$ for
ANOVA within activity declining groups <.05). Additionally, there were no significant differences in mean LM changes within the LM trajectories for the high-activitydeclining group (ANOVA within high activity group, $P=.81)$.

\section{Discussion}

In this descriptive study, we modeled trajectories of changes in PA and body composition over a nearly 7-year follow-up period with group based trajectory methods (GBTM). Three key findings emerged from these analyses. 

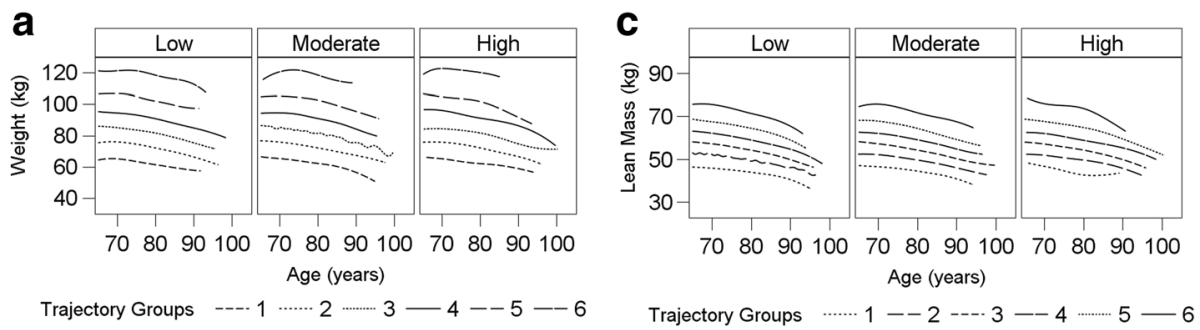

b

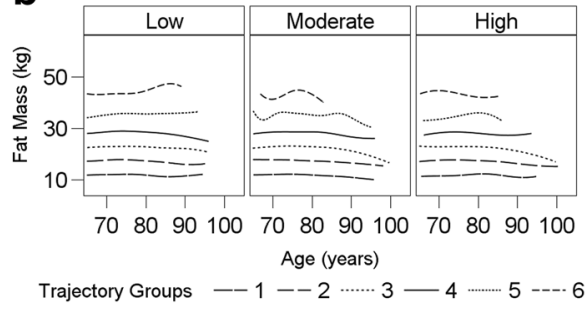

Fig. 3 Joint Trajectory modeling of PA trajectories across body weight (a), Total body fat mass (b), and, Lean mass (c) using BIC criteria $>410$ with $p<.05$

First, three discrete trajectories of PA change were identified among these older men, based primarily on baseline (regular) self-reported PA. All declined over the 7 year follow-up, however, the greatest declines were observed among men in the high-activity-declining group, compared to the moderate-activity- and low-activity-declining trajectories (both $P<.001$ ). Although the magnitude of the difference in change was modest it is important to recognize that men who report the highest PA levels at the outset of a longitudinal study may decrease PA much more than less active men over the course of the study. Nonetheless, these men still reported higher activity levels than the other two groups at subsequent visits. Second, greater declines in body weight and LM were observed among men with lower versus higher baseline body weight and LM, respectively. Joint-trajectory modeling showed that total body weight and LM declined, whereas fat mass levels were relatively unchanged among high activity- and low-activity-declining groups. In contrast, fat mass was significantly increased in three of the six moderate-activity-declining trajectories. Third, with-in group changes in body weight, fat mass and LM were associated with patterns of PA among low activity, and moderate activity declining men, but were not statistically different among high-activity-declining men.

Data on long-term PA changes in older men are scarce $[11,25,26]$. Previous studies of PA behavior in older adults have generally been cross-sectional $[14,27,28]$ or based on self-reported baseline PA levels only and have not captured an individual's change in activity over time. In the MrOS cohort, all trajectory modeling groups showed declines in PA levels, consistent with a previous MrOS report of decreased PA levels over a 5-year follow up, with the greatest PA declines attributed primarily to decreases in occupational, leisure, and household PA and poor health [24]. In contrast, de Souto et al. (2014) reported that total volume and duration of PA remained relatively stable in another cohort of older men; however, this was based on only 12- and 38 month follow ups [29].

To our knowledge, this is the first study to use latent class growth modeling analysis to describe, simultaneously, the relationship of changes in PA and changes in body-composition in older men. Examining the dynamic changes in PA as it relates to changes in body composition is particularly important in older adults, for whom the relation between PA and body composition may be the consequence of underlying poor health or chronic disease. Notably, a greater proportion of men in the lowactivity-declining reported having debilitating health issues versus those in the moderate- and high-activitydeclining groups (Table 1). Previous prospective studies showed a tendency toward lean mass loss and fat mass gain over time in healthy, weight-stable persons $[26,30]$. In the Women's Health Initiative (WHI) cohort of women aged 50-79, lean mass decreased across all age (decade) groups and all PA levels over about 6 years, whereas, fat mass change depended on both age and PA level, with higher PA levels attenuating fat gains in younger women (age 50-59), while fat loss was observed at older ages, regardless of PA level [9]. In contrast, in the MrOS cohort of men aged 65 and older, significant body weight and lean mass loss was observed in nearly all body weight and LM groups and across all PA change trajectories, whereas fat mass levels increased significantly among moderate-activity-declining groups, but remained relatively stable in high-activity- and lowactivity-declining trajectory groups $(P>.05)$ over nearly 7 years. Significant differences in the mean change of each body composition measure were 
$\sqrt{10}$

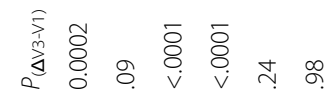

产

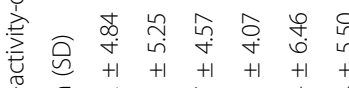

竞竞离

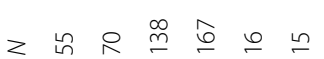

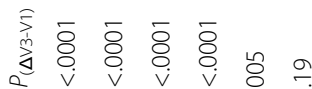

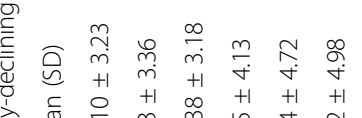

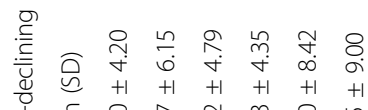

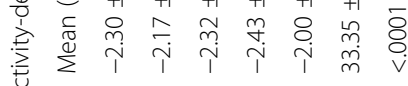

$\stackrel{5}{\geq}$

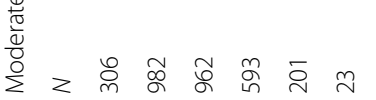

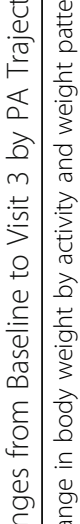

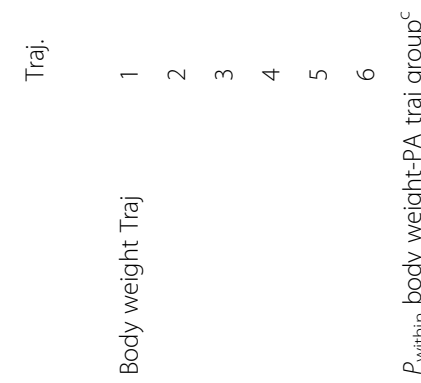

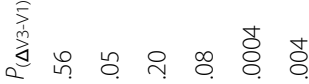

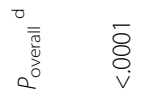

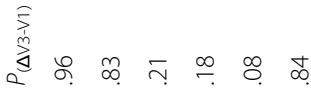

Q

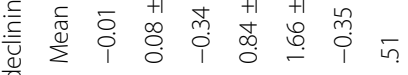

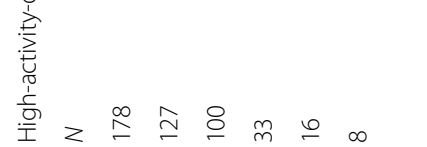

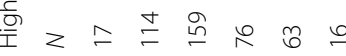

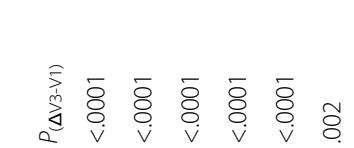

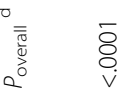

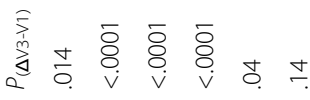

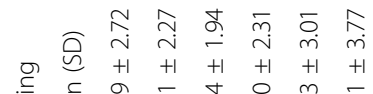

禀 $\sum_{\substack{i \\ \hline}}^{+}$
|⿱亠े禸

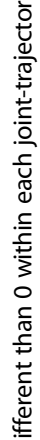


observed among men with in low-activity-and moderate-activity-declining PA groups, only (Table 2; Fig. 3), suggesting that changes in body weight, fat mass and LM were associated with patterns of PA among low-activity-, and moderate-activity declining men, but not among high-activity-declining men. Nonetheless, our findings of symmetry in the declining patterns of weight loss and LM further support the hypothesis that there is a failure to conserve lean mass with weight loss in old age $[4,11,25]$, whereas maintaining higher PA levels earlier in life and during aging may have a modest role in attenuating weight and lean mass declines in older men (Table 2, ANOVA within high activity group, both $P>0.05$ ), consistent with the results reported by previous investigators $[11,25]$.

\section{Strengths \& limitations}

These analyses included repeated PA measures in a large cohort of elderly men, allowing the capture of distinct, individual changes in PA engagement in older adults, and employed DXA to assess longitudinal body composition changes, using validated methods that correlate highly with other criterion methods [26]. As MrOS participants were predominantly healthy Caucasians, further investigation of patterns in PA-body composition changes in racial and ethnic minorities and men with specific health conditions, is warranted. Also, trajectory modeling is inherently limited in capturing individual variability and may lead to over-grouping with significant variation within PA trajectories. Additionally, PASE scores do not measure actual activity levels or the metabolic equivalent of a task or minutes of exercise; therefore, these results cannot be readily interpreted in relationship to national recommendations for PA in older adults. Corroborating the reported declines in PA with objective measures, to further understand PA in the context of dose (intensity, frequency and duration), may be warranted. Adjusting for intensity and mode of exercise to demarcate resistance training was not feasible in this study. Lastly, given the observational nature of the study, we cannot infer causality as to whether declines in loss of weight and lean mass were the result of declines in PA or confounded by other demographic, lifestyle or genetic factors affecting PA and body composition changes. Some of the groups identified, especially in the joint PA/body composition models, are very small and there is limited statistical power to draw conclusions about such groups.

\section{Conclusion}

The analyses from this longitudinal study of older men suggest that initial PA and body composition may differentiate PA and body composition changes over 6.9 years of follow-up.

\section{Additional file}

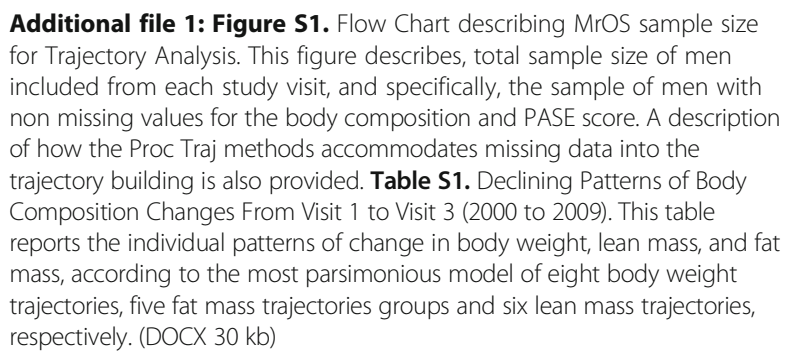

\section{Abbreviations}

BIC: Bayesian Information Criterion; DXA: Dual energy x-ray absorptiometry; GBTM: Group-based trajectory modeling; LM: Lean mass; MrOS: Osteoporotic Fractures in Men Study; PA: Physical activity; PASE: Physical Activity Scale for the Elderly; SD: Standard deviation; V: Visit

\section{Acknowledgments \\ None.}

\section{Funding}

The Osteoporotic Fractures in Men (MrOS) Study is supported by National Institutes of Health $(\mathrm{NIH})$ funding. The following institutes provide support: the National Institute of Arthritis and Musculoskeletal and Skin Diseases (NIAMS); the National Institute on Aging (NIA); the National Center for Research Resources (NCRR); and, NIH Roadmap for Medical Research under the following grant numbers: U01 AR45580, U01 AR45614, U01 AR45632, U01 AR45647, U01 AR45654, U01 AR45583, U01 AG18197, U01 AG027810, and

UL1 TR000128. Dr. Laddu was supported by the National Institutes of Health National Heart, Lung, and Blood Institute Cardiovascular Disease Prevention Fellowship (T32; grant HL007034).

This work was presented by Dr. Deepika R. Laddu at the 2016 International Conference on Frailty and Sarcopenia Research, April 28, 2016, Philadelphia, Pennsylvania.

\section{Availability of data and materials}

The datasets analyzed during the current study are available from the corresponding author and the MrOS coordinating center upon reasonable request.

\section{Authors' contributions}

DRL analyzed the data, interpreted the results and wrote the manuscript. DRL, PMC, MLS conceptualized the research question and designed the study. PMC provided consultation on methodology and helped with analysis interpretation. NP performed the statistical data analysis, and contributed to the interpretation of results. $\mathrm{ARH}, \mathrm{EO}$, and IM provided critical revisions to the manuscript. All authors approved the final version of the submitted and published manuscript and take responsibility for the integrity of the data and the accuracy of the data analysis. All authors contributed to the work equally.

\section{Competing interests}

The authors declare that they have no competing interests. Additionally, there are no conflicts of interest and there is nothing to disclose by any of the authors.

\section{Consent for publication}

Not applicable.

\section{Ethics approval and consent to participate}

The institutional review board at each center (i.e., Birmingham, AL; Minneapolis, MN; Palo Alto, CA; Pittsburgh, PA; Portland, OR; and San Diego, CA) approved the study protocol and written informed consent was obtained from all participants prior to participation. 


\section{Publisher's Note}

Springer Nature remains neutral with regard to jurisdictional claims in published maps and institutional affiliations.

\section{Author details}

'Department of Physical Therapy, University of Illinois at Chicago, Chicago, Illinois 60612, USA. ${ }^{2}$ California Pacific Medical Center Research Institute, San Francisco, California, USA. ${ }^{3}$ Department of Epidemiology and Biostatics, University of California, San Francisco, CA, USA. "Division of Endocrinology, Gerontology and Metabolism, Department of Medicine, Stanford University, Palo Alto, California, USA. ${ }^{5}$ Department of Medicine, Oregon Health \& Sciences University, Portland, OR, USA. ${ }^{6}$ Department of Epidemiology, University of Pittsburgh, Pittsburgh, PA, USA. ' Stanford Prevention Research Center, Department of Medicine, Stanford University, Stanford, California, USA

\section{Received: 28 December 2016 Accepted: 22 May 2017}

Published online: 05 June 2017

\section{References}

1. Dziura J. Mendes de Leon C, KasI S, DiPietro L: Can physical activity attenuate aging-related weight loss in older people? The Yale Health and Aging Study, 1982-1994. Am J Epidemiol. 2004;159(8):759-67.

2. Ensrud KE, Fullman RL, Barrett-Connor E, Cauley JA, Stefanick ML, Fink HA et al. Voluntary weight reduction in older men increases hip bone loss: the osteoporotic fractures in men study. J Clin Endocrinol Metab. 2005:90(4): 1998-2004.

3. Goodpaster BH, Park SW, Harris TB, Kritchevsky SB, Nevitt M, Schwartz AV, et al. The loss of skeletal muscle strength, mass, and quality in older adults: the health, aging and body composition study. J Gerontol A Biol Sci Med Sci. 2006;61(10):1059-64.

4. Newman AB, Lee JS, Visser M, Goodpaster BH, Kritchevsky SB, Tylavsky FA, et al. Weight change and the conservation of lean mass in old age: the Health, Aging and Body Composition Study. Am J Clin Nutr. 2005;82(4):872-8. quiz 915-876

5. Mitchell WK, Williams J, Atherton P, Larvin M, Lund J, Narici M. Sarcopenia, dynapenia, and the impact of advancing age on human skeletal muscle size and strength; a quantitative review. Front Physiol. 2012;3:260.

6. Cruz-Jentoft AJ, Baeyens JP, Bauer JM, Boirie Y, Cederholm T, Landi F, et al. Sarcopenia: European consensus on definition and diagnosis: Report of the European Working Group on Sarcopenia in Older People. Age Ageing. 2010;39(4):412-23.

7. Visser M, Goodpaster BH, Kritchevsky SB, Newman AB, Nevitt M, Rubin SM, et al. Muscle mass, muscle strength, and muscle fat infiltration as predictors of incident mobility limitations in well-functioning older persons. J Gerontol A Biol Sci Med Sci. 2005;60(3):324-33.

8. Forhan M, Gill SV. Obesity, functional mobility and quality of life. Best Pract Res Clin Endocrinol Metab. 2013:27(2):129-37.

9. Sims ST, Kubo J, Desai M, Bea J, Beasley JM, Manson JE, et al. Changes in physical activity and body composition in postmenopausal women over time. Med Sci Sports Exerc. 2013;45(8):1486-92.

10. Goodpaster BH, Chomentowski P, Ward BK, Rossi A, Glynn NW, Delmonico MJ, et al. Effects of physical activity on strength and skeletal muscle fat infiltration in older adults: a randomized controlled trial. Journal of applied physiology (Bethesda, Md : 1985). 2008;105(5):1498-503.

11. Hughes VA, Frontera WR, Roubenoff R, Evans WJ, Singh MA. Longitudinal changes in body composition in older men and women: role of body weight change and physical activity. Am J Clin Nutr. 2002;76(2):473-81.

12. Nelson ME, Rejeski WJ, Blair SN, Duncan PW, Judge JO, King AC, et al. Physical activity and public health in older adults: recommendation from the American College of Sports Medicine and the American Heart Association. Med Sci Sports Exerc. 2007;39(8):1435-45.

13. CDC/NCHS, National Health Interview Survey, 1997-2014, Sample Adult Core component, U.S. Department of Health and Human Services. Centers for Disease Control and Prevention: National Center for Health Statistics; 2014.

14. Matthews CE, Chen KY, Freedson PS, Buchowski MS, Beech BM, Pate RR, et al. Amount of time spent in sedentary behaviors in the United States, 2003-2004. Am J Epidemiol. 2008;167(7):875-81.

15. Di Pietro L, Dziura J, Blair SN. Estimated change in physical activity level $(\mathrm{PAL})$ and prediction of 5-year weight change in men: the Aerobics Center
Longitudinal Study. International journal of obesity and related metabolic disorders : journal of the International Association for the Study of Obesity. 2004;28(12):1541-7.

16. Williams PT, Wood PD. The effects of changing exercise levels on weight and age-related weight gain. International journal of obesity (2005). 2006; 30(3):543-51.

17. Genton L, Karsegard VL, Chevalley T, Kossovsky MP, Darmon P, Pichard C. Body composition changes over 9 years in healthy elderly subjects and impact of physical activity. Clinical nutrition (Edinburgh, Scotland). 2011; 30(4):436-42.

18. Nagin DS, Odgers CL. Group-based trajectory modeling in clinical research. Annu Rev Clin Psychol. 2010;6:109-38.

19. Jones BL, Nagin D. K R: A SAS Procedure Based on Mixture Models for Estimating Developmental Trajectories. Sociol Methods Res. 2001;29:374-93.

20. Orwoll E, Blank JB, Barrett-Connor E, Cauley J, Cummings S, Ensrud K, et al. Design and baseline characteristics of the osteoporotic fractures in men (MrOS) study-a large observational study of the determinants of fracture in older men. Contemporary clinical trials. 2005;26(5):569-85.

21. Blank JB, Cawthon PM, Carrion-Petersen ML, Harper L, Johnson JP, Mitson E, et al. Overview of recruitment for the osteoporotic fractures in men study (MrOS). Contemporary clinical trials. 2005;26(5):557-68.

22. Washburn RA, Smith KW, Jette AM, Janney CA. The Physical Activity Scale for the Elderly (PASE): development and evaluation. J Clin Epidemiol. 1993; 46(2):153-62.

23. Dinger MK, Oman RF, Taylor EL, Vesely SK, Able J. Stability and convergent validity of the Physical Activity Scale for the Elderly (PASE). The Journal of sports medicine and physical fitness. 2004;44(2):186-92.

24. Janney CA, Cauley JA, Cawthon PM, Kriska AM. Longitudinal physical activity changes in older men in the Osteoporotic Fractures in Men Study. J Am Geriatr Soc. 2010:58(6):1128-33.

25. Shiroma EJ, Sesso HD, Lee IM. Physical activity and weight gain prevention in older men. International journal of obesity (2005). 2012;36(9):1165-9.

26. Lee CG, Boyko EJ, Nielson CM, Stefanick ML, Bauer DC, Hoffman AR, et al. Mortality risk in older men associated with changes in weight, lean mass, and fat mass. J Am Geriatr Soc. 2011;59(2):233-40.

27. Martin KR, Koster A, Murphy RA, Van Domelen DR, Hung MY, Brychta RJ, et al. Changes in daily activity patterns with age in U.S. men and women: National Health and Nutrition Examination Survey 2003-04 and 2005-06. J Am Geriatr Soc. 2014;62(7):1263-71.

28. Kruger J, Carlson SA, Buchner D. How active are older Americans? Prev Chronic Dis. 2007:4(3):A53.

29. de Souto BP, Ferrandez AM. Static or dynamic predictors of physical activity (PA)? A tracking study based on 12- and 38-month follow-ups in older adults. Arch Gerontol Geriatr. 2014:59(2):326-30.

30. Gallagher D, Ruts E, Visser M, Heshka S, Baumgartner RN, Wang J, et al. Weight stability masks sarcopenia in elderly men and women. Am J Phys Endocrinol Metab. 2000;279(2):E366-75

\section{Submit your next manuscript to BioMed Central and we will help you at every step:}

- We accept pre-submission inquiries

- Our selector tool helps you to find the most relevant journal

- We provide round the clock customer support

- Convenient online submission

- Thorough peer review

- Inclusion in PubMed and all major indexing services

- Maximum visibility for your research

Submit your manuscript at www.biomedcentral.com/submit
) Biomed Central 